\title{
ITENS LEXICAIS ESTRANGEIROS COMO TRAÇO ESTILÍSTICO EM THINGS FALL APART: UM ESTUDO EM CORPUS PARALELO
}

\author{
FOREIGN LEXICAL ITEMS AS STYLISTIC FEATURE IN THINGS FALL APART: A \\ STUDY IN PARALLEL CORPUS
}

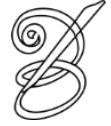 \\ Cristina Lazzerini de Souza ${ }^{1}$ \\ Mestranda em Estudos Linguísticos \\ (POSLIN - UFMG - Belo Horizonte - Brasil) \\ crislazzerini@gmail.com
}

\begin{abstract}
Resumo: Este artigo investiga o uso de itens lexicais estrangeiros (ILEs) como traço estilístico em duas traduções para o português de Things Fall Apart. Utiliza a estilística tradutória para identificar e descrever padrões de uso de ILEs, empregando procedimentos e usando ferramentas da linguística de corpus para buscar os dados estatísticos gerais do corpus e os dados referentes ao uso de ILEs. Resultados parciais do estudo identificam mudanças estilísticas ocorridas nas traduções, as quais sugerem que os tradutores empregam diferentes estratégias para tratar os ILEs.
\end{abstract}

Palavras-chave: itens lexicais estrangeiros, estilo da tradução, corpus paralelo, Things Fall Apart.

Abstract: This article investigates the use of foreign lexical items (FLIs) as a stylistic feature in two translations into Portuguese of Things Fall Apart. It applies translational stylistics to identify and describe the patterns of use of FLIs, employing procedures and using tools of corpus linguistics to gather the statistic data from the corpus and the data about the use of FLIs. Partial results of the study identify stylistic shifts in the translation, which suggest the translators use different strategies to deal with the FLIs.

Keywords: foreign lexical items, style of translation, parallel corpus, Things Fall Apart.

\section{Introdução}

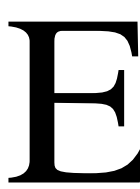

ste estudo exploratório insere-se no âmbito dos estudos de estilo do texto traduzido (TT), usa um corpus paralelo em inglês-português, e utiliza metodologia da etapa descritiva da estilística tradutória para a identificação e descrição das mudanças e padrões de usos dos ILEs. Difere de outros estudos que investigam ILEs como FrankenbergGarcia (2005) que, embora trabalhe com o par de idiomas inglês-português, não investiga os ILEs como traço de estilo, e Saldanha (2011a) que, embora investigue ILEs como traço de estilo, enfoca o estilo do tradutor. O objetivo do trabalho é encontrar padrões de uso diferenciados dos ILEs nas traduções e seu provável efeito no significado dos TTs. Para 
atingir o objetivo, o aporte teórico é o da estilística tradutória. A metodologia consiste de procedimentos de análise de corpus com o uso de ferramentas de programa de análise lexical.

Este artigo possui quatro seções. A primeira apresenta uma revisão das teorias que embasam esta pesquisa; a segunda apresenta o corpus e os procedimentos metodológicos empregados; a terceira apresenta e discute os resultados; e a quarta tece considerações a respeito da pesquisa.

\section{Revisão da Literatura}

A estilística tradutória é uma metodologia para análise de estilo de TTs proposta por Malmkjaer (2003, 2004). Vai além do estudo de estilo, que se ocupa das "regularidades de ocorrências, consistentes e estatisticamente significantes, no texto, de certos itens e estruturas ou tipos de estruturas entre outros oferecidos pela língua como um todo” (MALMKJAER e CARTER 2002, apud MALMKJAER 2003, p. 38)², por usar um nível supra-descritivo semântico; e da estilística de textos literários monolíngues, por tomar como ponto de partida os TTs em relação aos TFs. A estilística tradutória encarrega-se de explicar a maneira - como

- e o motivo pelo qual - por que - o escritor construiu o texto de determinada forma, tendo fatores extralinguísticos como limitadores de sua liberdade.

Malmkjaer (2004) aponta características das traduções enquanto textos mediados que devem ser levadas em conta na análise da estilística tradutória:

\footnotetext{
um texto mediado é afetado pela interpretação do texto original feita pelo mediador; a mediação através de tradução sempre tem um propósito; o propósito estabelecido para a tradução pode ser diferente daquele do texto original; a audiência da tradução é quase sempre diferente da audiência do texto original (MALMKJAER 2004, p.16) ${ }^{3}$
}

A estilística tradutória considera estilo como atributo textual, sem deixar de considerar a presença do tradutor nos textos. A análise que segue essa metodologia é dividida em duas etapas. A primeira tem como objetivo descrever o estilo, "como o texto significa o que significa” (MALMKJAER, 2003, p. 39, grifo da autora) ${ }^{4}$. Isso implica descrever os padrões de mudanças nos TTs utilizando categorias de análise dos estudos linguísticos. A segunda etapa consiste em explicar "por que, dado o texto fonte, a tradução foi construída de tal forma a vir significar o que significa” (MALMKJAER, 2003, p. 39, grifo da autora) ${ }^{5}$. Isso implica em tentar encontrar evidências para a motivação das escolhas que levam ao estilo diferenciado. O presente estudo, por seu escopo limitado, baseia-se apenas na primeira etapa da metodologia. 
Dentre os estudos sobre ILEs em tradução utilizando metodologia de LC no par de idiomas inglês/português encontram-se Frankenberg-Garcia (2005), afiliado aos ETBC, Saldanha (2011a), afiliado aos estudos de estilo.

Frankenberg-Garcia (2005) trabalhou com um corpus de estudo comparável bilíngue, a partir do COMPARA. Teve como objetivo comparar o uso de empréstimos em textos de ficção traduzidos e não traduzidos, enfocando a frequência de uso e a distribuição dos empréstimos por língua. Para encontrar os empréstimos nos textos, utilizou o recurso busca complexa do COMPARA, que encontra automaticamente palavras em línguas diferentes daquela do texto que foram marcadas tipograficamente - principalmente com itálicos. Não considerou como empréstimos títulos de livros e filmes, e nomes de pessoas, lugares, produtos e organizações em outra língua. As expressões contendo mais de uma palavra estrangeira (PE) foram consideradas um único empréstimo. Os resultados em FrankenbergGarcia (2005) mostram que a tendência maior, em ambas as línguas, é preservar os empréstimos existentes nos TFs; tende-se, ainda, a acrescentar alguns empréstimos e, em menor proporção, omiti-los.

Saldanha (2011a) trabalhou com dois corpora, um de traduções de Peter Bush (PB) e outro de traduções de Margaret Jull Costa (MJC) e teve como objetivo identificar traços estilísticos que pudessem ser atribuídos a cada um dos tradutores investigados. Partiu de uma pesquisa anterior - Saldanha 2005 - que havia constatado que uma das funções básicas da tipologia dos itálicos é a diferenciação para marcar PEs. Não incluiu nomes próprios, títulos e citações em línguas estrangeiras. Adotou a terminologia ILE, uma vez que considerou como uma única unidade as ocorrências com mais de uma PE representando uma única ideia ou conceito. Saldanha (2011a) constatou que a maioria dos ILEs italicizados nos TTs pertence à língua dos TFs. Concluiu que PB utiliza mais empréstimos para lidar com itens culturais específicos do que MJC, a qual evita utilizar itens lexicais com os quais seus leitores não estejam familiarizados e, se o faz, procura facilitar seu entendimento através de informação contextual. Atribui os padrões de uso dos ILEs a escolhas estilísticas pessoais dos tradutores e sugere, a partir da análise de dados metatextuais, que essas escolhas se devem principalmente ao papel que cada tradutor se atribui enquanto mediador intercultural, em relação a seus leitores. 


\section{Metodologia}

\subsection{O Corpus}

O corpus utilizado nesta pesquisa é paralelo, composto pela obra Things Fall Apart, do escritor nigeriano Chinua Achebe, edição de 1994; sua tradução para o português brasileiro, O Mundo se Despedaça, edição de 1983, realizada por Vera Queiróz da Costa e Silva; e sua tradução para o português europeu Quando Tudo se Desmorona, edição de 2008, realizada por Eugénia Antunes e Paulo Rêgo. O corpus possui 156.000 palavras, portanto, considerado de pequena dimensão. Foi selecionado para esta pesquisa por ser um subcorpus do Corpus de Estilo da Tradução - ESTRA - (MAGALHÃES, 2014) do LETRA/UFMG e porque espera-se encontrar na obra de Achebe o uso recorrente de ILEs, e, portanto, estratégias diferenciadas na tradução desses itens.

Achebe é considerado um dos expoentes da literatura africana. Diferente de outros escritores africanos que priorizaram suas línguas-maternas, o escritor pós-colonial nigeriano adotou a língua do colonizador para transmitir as experiências de seu povo. Achebe é creditado por ter criado o New English, que mistura a oralidade africana com a forma literária inglesa. Uma de suas estratégias é a utilização de palavras e expressões em ibo para a representação cultural da experiência africana.

\subsection{Procedimentos de preparação e análise do corpus}

Os arquivos do corpus desta pesquisa são TFA_Achebe, TFA_Antunes_Rego e TFA_CostaeSilva_83, nomeados segundo os critérios do ESTRA. A preparação dos textos incluiu a colocação de etiquetas para as ocorrências de itálicos <i>, porém, os ILEs não possuem marcação específica no corpus.

As metodologias de pesquisa baseadas em corpus envolvem a coleta de dados numéricos e/ou estatísticos que requerem a intervenção e interpretação humana, desde a contagem dos ILEs identificados e sua distribuição, facilitados pelo formato digital dos textos e pelas ferramentas de corpus, até a interpretação em relação ao tema do estilo.

Neste estudo, emprega-se o programa de análise lexical WST, que possui três ferramentas - WordList, KeyWords e Concord - e doze utilitários. Para a coleta dos dados estatísticos gerais, utiliza a ferramenta WordList que gera as listas de palavras para cada um dos textos do corpus, com o número de itens (tokens) e formas (types) e a razão entre eles.

Este estudo parte da investigação dos itálicos com o objetivo de encontrar ILEs, justificando-se pelas pesquisas realizadas em Frankenberg-Garcia (2005) e Saldanha (2011a). 
Adota, com Saldanha (2011a), a terminologia ILEs. Seguindo Frankenberg-Garcia (2005), num primeiro momento, a decisão do que é considerado ILE é pré-determinada por quem o marcou tipograficamente. Num segundo momento, itens lexicais não marcados tipograficamente são considerados estrangeiros se em língua diferente daquela do texto em que se encontram.

A ferramenta Concord do WST é utilizada para encontrar os itens lexicais italicizados, buscando-se pela etiqueta $<\mathrm{i}>$. A ferramenta gera as linhas de concordâncias desses itens, com o número total de itálicos em cada texto. A seguir, é feita uma análise para descartar as ocorrências de itálicos que não marcam ILEs, contabilizando-se apenas aquelas em que os itálicos marcam ILEs.

Na próxima etapa, as linhas de concordância de cada texto contendo ILEs são alinhadas. Para tanto, as ocorrências de ILEs são colocadas na ordem em que aparecem no texto, utilizando a opção concordance sort do Concord. As linhas são expandidas e, com o cotexto selecionado, são recortadas e coladas em um quadro com três colunas de linhas de concordância equivalentes. Ao final desse processo, observa-se que há várias linhas de concordância sem suas correspondentes, portanto, outras buscas são realizadas para encontrálas. Uma vez completo o quadro, calcula-se o total de ILEs para cada texto.

O próximo passo consiste em observar o que ocorre com os ILEs nos TTs, classificando-os em quatro categorias, conforme mostra o Quadro 1.

Quadro 1 - Categorias de estratégias para tratamento dos ILEs

\begin{tabular}{|l|l|l|l|}
\hline \multicolumn{1}{|c|}{ Trazidos do TF } & \multicolumn{1}{c|}{ Traduzidos } & \multicolumn{1}{c|}{ Omitidos } & \multicolumn{1}{c|}{ Acrescentados } \\
\hline O tradutor leva para & O tradutor utiliza um & O tradutor descarta o & O tradutor utiliza um \\
o TT o ILE utilizado & item na língua alvo \\
pelo autor do TF. & $\begin{array}{l}\text { equivalente ao ILE } \\
\text { utilizado pelo autor } \\
\text { do TF. }\end{array}$ & autor do TF. & ILE que não está no \\
& & \\
\hline
\end{tabular}

Finalmente, contabiliza-se o número de ILEs em cada uma das categorias e procura-se por padrões de uso dos ILEs. Os padrões identificados nos TTs são comparados entre si e em relação ao TF. As mudanças ocorridas e suas implicações no estilo dos TTs são descritas. 


\section{Apresentação e discussão dos resultados}

O primeiro procedimento de análise foi realizado com o suporte da ferramenta WordList. Os dados estatísticos gerais do corpus obtidos com as listas de palavras são apresentados na Tabela 1.

Tabela 1 - Dados estatísticos do corpus

\begin{tabular}{|l|l|l|l|l|}
\hline & $\begin{array}{l}\text { Itens } \\
\text { (tokens) }\end{array}$ & $\begin{array}{l}\text { Formas } \\
\text { types) }\end{array}$ & $\begin{array}{l}\text { Razão } \\
\text { forma/item } \\
\%\end{array}$ & $\begin{array}{l}\text { Razão forma/item } \\
\text { padronizada } \\
\%(1.000)\end{array}$ \\
\hline TFA_Achebe & 51.541 & 4.421 & 8,58 & 39,03 \\
\hline TFA_Antunes_Rego & 50.550 & 6.926 & 13,70 & 45,73 \\
\hline TFA_CostaeSilva_83 & 53.909 & 7.375 & 13,68 & 47,29 \\
\hline
\end{tabular}

Dos resultados depreende-se que, quanto ao número de itens, TFA_Antunes_Rego é 1,93\% menor do que o TF e TFA_CostaeSilva é 4,59\% maior do que o TF. O fato de a tradução para o português europeu ter menos itens do que o original não pode ser atribuído à diferença entre língua-fonte e meta levantada por Klaudy and Károly (apud SALDANHA, 2005, p. 76), segundo a qual o fato de as línguas serem mais analíticas ou mais sintéticas influi no tamanho dos textos fonte e meta, pois as línguas analíticas são consideradas mais econômicas, e nas línguas em questão, o inglês é considerado mais analítico do que o português.

A princípio, o menor número de itens de TFA_Antunes_Rego não confirma a explicitação, proposta por Baker (1993) como um universal de tradução e considerada por Toury (apud Baker, 1993, p. 244) típica de todos os eventos mediados. Uma investigação do corpus é necessária para confirmar essa hipótese ou atribuir o resultado à implicitação, não sendo este o propósito deste estudo. Por outro lado, TFA_CostaeSilva_83, com maior número de itens do que o original, apontaria para explicitação, tendência observada a partir das linhas de concordância coletadas, como mostram os exemplos do Quadro 2. Outro universal de tradução ao qual um maior número de itens pode ser atribuído é a tendência a evitar 
repetições que podem ter significado literário nos TTs (Baker 1993), uma vez que reformulações podem tornar os TTs mais longos.

A razão forma/item de ambos os TTs é maior do que a do TF, o que pode ser um indicativo de que os tradutores usam uma maior variedade de vocabulário que o autor. Observa-se que quanto à razão bruta, os números variam pouco de uma tradução para outra; de acordo com a razão normalizada, a variedade de vocabulário utilizada por Costa e Silva é 1,56\% maior do que a empregada por Antunes e Rêgo. Em relação ao TF, a variedade de vocabulário de Antunes e Rêgo é 6,7\% maior e a de Costa e Silva 8,26\% maior. Deve-se ter cautela com esta interpretação, já que há diferenças nos dois sistemas linguísticos no que tange sua morfologia.

O Quadro 2 apresenta exemplos em que apenas a tradutora explicita os ILEs.

Quadro 2 - Exemplos de explicitação de ILEs em TTs

\begin{tabular}{|l|l|l|}
\hline \multicolumn{1}{|c|}{ TFA_Achebe } & \multicolumn{1}{|c|}{ TFA_Antunes_Rego } & \multicolumn{1}{|c|}{ TFA_CostaeSilva_83 } \\
\hline $\begin{array}{l}\text { the faint beating of the } \\
\text { ekwe. }\end{array}$ & o débil tocar do ekwe. & $\begin{array}{l}\text { o vago bater do } e k w e, \text { o tambor } \\
\text { de madeira. }\end{array}$ \\
\hline "It is iba," & -- É iba -- & -- É a iba, a malária -- \\
\hline
\end{tabular}

Observa-se nos exemplos do Quadro 2 que tanto em TFA_Achebe quanto em TFA_Antunes_Rego, os itens ekwe e iba foram incorporados aos textos sem nenhuma explicação. Em TFA_CostaeSilva_83, no entanto, a tradutora acrescenta um aposto explicativo. Esse tipo de mudança facilita a leitura do texto e constitui uma estratégia diferente daquela empregada pelo autor do TF e os tradutores Antunes e Rêgo.

A Tabela 2, a seguir, mostra os resultados obtidos com a contabilização dos itálicos, por meio do Concord. 
Tabela 2 - Dados quantitativos de itálicos do corpus

\begin{tabular}{|l|l|l|l|}
\hline & $\begin{array}{l}\text { Total de } \\
\text { ocorrências } \\
\text { de itálicos }\end{array}$ & $\begin{array}{l}\text { Ocorrências de itálicos } \\
\text { marcando ILEs } \\
\text { (com repetições) }\end{array}$ & $\begin{array}{l}\text { Ocorrências de itálicos } \\
\text { marcando ILEs } \\
\text { (sem repetições) }\end{array}$ \\
\hline TFA_Achebe & $\begin{array}{l}229 \\
100 \%\end{array}$ & $\begin{array}{l}216 \\
94,32 \%\end{array}$ & $\begin{array}{l}46 \\
20,08 \%\end{array}$ \\
\hline TFA_Antunes_Rego & 243 & 221 & 47 \\
$100 \%$ & $90,94 \%$ & $19,34 \%$ \\
\hline TFA_CostaeSilva_83 & 298 & 284 & 49 \\
& $100 \%$ & $95,30 \%$ & $16,44 \%$ \\
\hline
\end{tabular}

O número total de itálicos para cada texto foi gerado pela ferramenta Concord quando se buscou pela etiqueta $<\mathrm{i}>$. Nota-se que ambos os TTs apresentam maior número de ocorrências de itálicos do que o TF. Em TFA_Antunes_Rego, houve acréscimo de 6,11\% do número total de itálicos em relação a TFA_Achebe; e em TFA_CostaeSilva esse acréscimo 46 foi de 30,13\% com relação ao original.

O cálculo do número total de ILEs marcados por itálicos foi feito descartando-se os itálicos usados com outras funções. Em TFA_Achebe, as ocorrências de itálicos usados com outras funções foram 13, em TFA_Antunes_Rego, 22, e emTFA_CostaeSilva_83, 14. Em TFA_Antunes_Rego, 221 ILEs italicizados significam um aumento de 2,31\% em relação ao TF; este aumento é ainda maior em TFA_CostaeSilva_83, em que 284 ILEs significam $31,48 \%$.

Embora tenha havido aumento tanto do número total de itálicos, quanto do número de itálicos marcando ILEs, em ambos os TTs em relação ao TF, a proporção do número de itálicos marcando ILEs em relação ao número total de itálicos em cada TT nem sempre aumentou em relação ao original. Em TFA_Achebe, 94,32\% das ocorrências de itálicos marcam ILEs; esta porcentagem cai para 90,94\% em TFA_Antunes_Rego e aumenta para 95,30\% em TFA_CostaeSilva_83. Esses números apontam para diferentes estratégias de uso de itálicos por parte dos tradutores, mas, de qualquer forma, são condizentes com os resultados de Saldanha (2005), confirmando a tendência da maioria dos empregos de itálicos ser para diferenciação de ILEs.

Quanto ao número de ILEs distintos marcados por itálicos, percebe-se que em TFA_Antunes_Rego, houve acréscimo de um ILE marcado por itálico, a palavra ewuro, que 
não existe em TFA_Achebe. Já Costa e Silva acrescentou itálicos a três ILEs distintos - Afo, compound e egusi - todas as três palavras se encontram em TFA_Achebe, no entanto, sem marcação de itálicos. A palavra compound não é ILE no TF e, a princípio, essa palavra inglesa poderia ter equivalente em português, mas a tradutora optou por trazê-la do TF, destacando-a com itálicos. Esses resultados indicam mudanças estilísticas realizadas nos TTs e requerem investigação quanto aos prováveis efeitos de tais escolhas nos TTs. O Quadro 3, a seguir, mostra os exemplos em que ocorrem tais ILEs.

Quadro 3 - Exemplos de acréscimo de itálicos e de ILEs

\begin{tabular}{|l|l|l|}
\hline \multicolumn{1}{|c|}{ TFA_Achebe } & \multicolumn{1}{|c|}{ TFA_Antunes_Rego } & \multicolumn{1}{|c|}{ TFA_CostaeSilva_83 } \\
\hline bitter-leaf soup & $\begin{array}{l}\text { sopa de folhas amargas de } \\
\text { ewuro }\end{array}$ & sopa de folha-amarga \\
\hline on every other Afo day & dia Afo sim dia Afo não & nos dias de Afo \\
\hline outside their compound & do lado de fora do recinto & quase ao lado do compound \\
\hline egusi soup & sopa de egussi & sopa de egusi \\
\hline
\end{tabular}

A Tabela 3 apresenta os dados quantitativos da classificação das ocorrências de ILEs nos TTs realizada com o suporte das linhas de concordância. Foram encontrados 234 ILEs no TF, 230 no TT para o português europeu e 284 no TT para o português brasileiro. Observa-se que o preenchimento das lacunas do quadro de linhas de concordância alinhadas acarretou a inclusão de ILEs que não haviam sido encontrados pela busca de itálicos. Assim sendo, o total de ILEs em TFA_Achebe e TFA_Antunes_Rego é maior que o número total de ILEs marcados por itálicos (Tabela 2, acima). Em TFA_CostaeSilva, esses números são iguais, indicando que todos os ILEs encontrados estavam marcados por itálicos. 
Tabela 3 - Resultados quantitativos da classificação dos ILEs nos TTs

\begin{tabular}{|c|c|c|c|c|c|}
\hline & $\begin{array}{l}\text { Total } \\
\text { de ILEs }\end{array}$ & $\begin{array}{l}\text { Número } \\
\text { de ILEs } \\
\text { trazidos } \\
\text { do TF }\end{array}$ & $\begin{array}{l}\text { Número } \\
\text { de ILEs } \\
\text { traduzidos }\end{array}$ & $\begin{array}{l}\text { Número } \\
\text { de ILEs } \\
\text { omitidos }\end{array}$ & $\begin{array}{l}\text { Número } \\
\text { de ILEs } \\
\text { acrescentados }\end{array}$ \\
\hline TFA_Achebe & 234 & & & & \\
\hline $\begin{array}{l}\text { TFA_Antunes_R } \\
\text { ego }\end{array}$ & $\begin{array}{l}230 \\
100 \%\end{array}$ & $\begin{array}{l}216 \\
93,91 \%\end{array}$ & $\begin{array}{l}13 \\
5,65 \%\end{array}$ & $\begin{array}{l}3 \\
1,30 \%\end{array}$ & $\begin{array}{l}14 \\
6,08 \%\end{array}$ \\
\hline $\begin{array}{l}\text { TFA_CostaeSilva } \\
\text { _83 }\end{array}$ & $\begin{array}{l}284 \\
100 \%\end{array}$ & $\begin{array}{l}222 \\
78,16 \%\end{array}$ & $\begin{array}{l}6 \\
2,11 \%\end{array}$ & $\begin{array}{l}6 \\
2,11 \%\end{array}$ & $\begin{array}{l}62 \\
21,83 \%\end{array}$ \\
\hline
\end{tabular}

Percebe-se que TFA_Antunes_Rego possui quatro ILEs a menos do que TFA_Achebe e que TFA_CostaeSilva_83 possui 50 a mais. Em relação ao TF, TFA_Antunes_Rego tem 98,29\% de ILEs e TFA_CostaeSilva_83 121,36\%. A grande diferença entre TFA_CostaeSilva_83 e os outros dois textos se explica pelo item compound, com 53 ocorrências, e que a tradutora trouxe do TF, marcando-as com itálicos. Portanto, esse item não é palavra estrangeira no TF, nem em TFA_Antunes_Rego, em que foi traduzido por recinto, casa e redil, mas constitui ILE acrescido em TFA_CostaeSilva_83. A porcentagem de itens lexicais acrescentados em TFA_CostaeSilva_83 é de 21,83\% e em TFA_Antunes_Rego, $6,08 \%$.

Os números da Tabela 3 mostram que a maioria dos ILEs foi trazido do TF em ambas as traduções, 93,91\% em TFA_Antunes_Rego e 78,16\% em TFA_CostaeSilva_83. Nota-se que Antunes e Rego traduzem mais ILEs que Costa e Silva. Em TFA_Antunes_Rego, 5,65\% dos ILEs foram traduzidos; as 13 ocorrências correspondem a duas palavras - egussi e fufu a primeira com duas ocorrências e a segunda com 11. Em TFA_CostaeSilva_83, as 6 ocorrências são todas de agogô, tradução de ogene, e representam 2,11\% dos ILEs. Omissão é a estratégia menos utilizada por Antunes e Rego. Antunes e Rego omitem 1,30\%, e Costa e Silva 2,11\%. Costa e Silva traduz e omite a mesma quantidade, 2,11\%.

Exemplos das ocorrências de compound podem ser vistos no Quadro 4, a seguir. 
Quadro 4 - Exemplos de ocorrências de compound e equivalentes

\begin{tabular}{|l|l|l|}
\hline \multicolumn{1}{|c|}{ TFA_Achebe } & \multicolumn{1}{|c|}{ TFA_Antunes_Rego } & \multicolumn{1}{|c|}{ TFA_CostaeSilva_83 } \\
\hline $\begin{array}{l}\text { She did not return to } \\
\text { Okonkwo's compound }\end{array}$ & $\begin{array}{l}\text { Só regressou a casa de } \\
\text { Okonkwo }\end{array}$ & $\begin{array}{l}\text { A mulher não voltou ao } \\
\text { compound de Okonkwo }\end{array}$ \\
\hline $\begin{array}{l}\text { The goat was then led back } \\
\text { to the inner compound. }\end{array}$ & $\begin{array}{l}\text { A cabra foi então conduzida } \\
\text { de volta ao redil. }\end{array}$ & $\begin{array}{l}\text { A cabra tornou a ser levada } \\
\text { para o interior do compound. }\end{array}$ \\
\hline $\begin{array}{l}\text { Nwoye turned round to walk } \\
\text { into the inner compound }\end{array}$ & $\begin{array}{l}\text { Nwoye virara-se para avançar } \\
\text { para o recinto }\end{array}$ & $\begin{array}{l}\text { Nwoye deu meia-volta, } \\
\text { fazendo menção de se } \\
\text { encaminhar para o interior do } \\
\text { compound; }\end{array}$ \\
\hline $\begin{array}{l}\text { Maduka vanished into the } \\
\text { compound like lightning. }\end{array}$ & $\begin{array}{l}\text { Maduka desapareceu no meio } \\
\text { do recinto como um raio. }\end{array}$ & $\begin{array}{l}\text { Maduka saiu como um raio } \\
\text { na direção do terreiro. }\end{array}$ \\
\hline
\end{tabular}

Os três primeiros exemplos do Quadro 4 mostram que Antunes e Rego traduzem o item compound por casa, redil e recinto, enquanto Costa e Silva traz o ILE do TF. Porém, o último exemplo mostra que Costa e Silva não utilizou a mesma estratégia para todas as ocorrências de compound do TF, mas traduziu-a algumas vezes por terreiro.

Exemplos de ILEs traduzidos podem ser vistos no Quadro 5, a seguir.

Quadro 5 - Exemplos de itens lexicais traduzidos

\begin{tabular}{|l|l|l|}
\hline \multicolumn{1}{|c|}{ TFA_Achebe } & \multicolumn{1}{|c|}{ TFA_Antunes_Rego } & \multicolumn{1}{c|}{ TFA_CostaeSilva_83 } \\
\hline $\begin{array}{l}\text { There was foo-foo and yam } \\
\text { pottage, egusi soup }\end{array}$ & $\begin{array}{l}\text { Havia fufu e caldo de inhame, } \\
\text { sopa de egussi }\end{array}$ & $\begin{array}{l}\text { Havia foo-foo e sopa de } \\
\text { inhame, sopa de egusi }\end{array}$ \\
\hline He played on the ogene. & Tocava ogene. & Tocava o agogô. \\
\hline
\end{tabular}

Observa-se nos exemplos do Quadro 5 que Antunes e Rego traduziram os itens foo-foo e egusi enquanto Costa e Silva os trouxe do TF e os italicizou. Já o item ogene foi trazido do TF por Antunes e Rego e traduzido por Costa e Silva. Constata-se, portanto, que os tradutores tomaram decisões diferentes quanto aos itens a serem traduzidos e não foi a ausência de item equivalente na língua-fonte o fator determinante de tais decisões. 
Diferente de Saldanha (2005), a presente pesquisa encontrou um grande número de ILEs nos TTs, um total de 514 ocorrências num corpus de 104.459 itens. Esse número, que reflete a quantidade de ILEs presentes no TF, possibilita a observação do objeto de estudo. Neste estudo, todos ILEs, exceto um, são de uma terceira língua, da cultura retratada nos textos, e não na língua-fonte do TF, como ocorre com a maioria dos ILEs nos estudos anteriores.

Quanto às estratégias adotadas pelos tradutores para o tratamento dos ILEs constatouse que os ILEs foram trazidos do TF, traduzidos e omitidos nos TTs. Também se verificou que, nas duas traduções, foram acrescentados ILEs; observaram-se acréscimos de ocorrências de repetições de ILEs presentes no original, de ocorrências de ILE distinto que não se encontrava no original, e de item lexical não estrangeiro no TF, transformado em estrangeiro no TT. Como em Frankenberg-Garcia (2005), a estratégia mais utilizada nos TTs é a de trazer os ILEs do TF.

Outra constatação feita é que, embora a maioria dos ILEs tenha sido trazida do TF, a forma como os tradutores escolheram fazê-lo varia. Ocorrências retiradas de 50 TFA_CostaeSilva_83 indicam uma tendência à explicitação que precisa ser melhor investigada. A hipótese é que a tradutora faz uso consciente de uma estratégia de explicitação para tornar o texto mais fluente para seus leitores e que isso afeta o estilo do texto. Em consonância com os estudos anteriores, esta pesquisa constatou que a maioria dos ILEs presentes em todos os textos estão italicizados.

\section{Considerações finais}

Este estudo exploratório, que investigou o uso de ILEs como traço estilístico em duas traduções de Things Fall Apart para o português com base em estudos de estilo da tradução e a metodologia de corpus, identificou e classificou os ILEs de acordo com as estratégias utilizadas pelos tradutores para o tratamento desses itens, mostrando escolhas tradutórias proeminentes, não restritas pelas escolhas do autor do TF ou pelo sistema linguístico meta. Validou o emprego da metodologia de corpus para o estudo do estilo de textos literários e vem contribuir para a construção do conhecimento sobre estilo da tradução.

Reconhece-se que os dados aqui apresentados são preliminares e parciais. A pesquisa exploratória identificou apenas alguns dos padrões de mudanças sofridas pelos ILEs no processo tradutório. Porém, essas mudanças fornecem subsídios para a próxima etapa de 
pesquisa, na qual serão investigadas, do ponto de vista linguístico e de estratégias tradutórias, buscando suas possíveis motivações e seus efeitos nos TTs.

\section{REFERÊNCIAS BIBLIOGRÁFICAS}

ACHEBE, Chinua. Morning Yet on Creation Day. New York: Anchor Press/ Doubleday, 1975, p. 91-103.

O mundo se despedaça. Trad. Vera Queiroz da Costa e Silva. São Paulo: Editora

Ática S. A., 1983.

. Things Fall Apart. New York: Anchor Books, [1958]1994.

Quando tudo se desmorona. Trad. Eugénia Antunes e Paulo Rêgo. Lisboa:

Mercado de Letras Editores, 2008.

ANDERSON, Wendy.; CORBETT, John. Exploring English with online Corpora.

Palgrave: Macmillan, 2009, p. 21-44.

BAKER, Mona. Corpus Linguistics and Translation Studies: Implications and Applications. In: BAKER, Mona; FRANCIS, Gill; TOGNINI-BONELLI, Elena (eds.) Text and Technology: In honour of John Sinclair. Amsterdam \& Philadelphia: John Benjamins Publishing Company, 1993, p. 233- 250.

. Corpus-based translation studies: The challenges that lie ahead. In: SOMERS, Harold (Ed.), Terminology, LSP and Translation: Studies in language engineering in honour of Juan C. Sager. Amsterdam \& Philadelphia: John Benjamins Publishing Company, 1996, p.175-187.

BERBER-SARDINHA, Tony. Pesquisa em Linguística de Corpus com WordSmith Tools. 2006.

FRANKENBERG-GARCIA, Ana. A corpus based study of loan words in original and translated texts. Lisboa: ISLA, 2005.

MAGALHÃES, Célia. ESTRA: um corpus para o estudo do estilo da tradução. Cadernos de Tradução, Florianópolis, n. 34, p. 248-271, jul./dez. 2014.

MALMKJAER, Kristen. What happened to God and the angels: an exercise in translational stylistics. Target, v. 15. Amsterdam, 2003. p. 37-58.

Translational stylistics: Dulcken's translations of Hans Christian Andersen. Language and Literature. London, v. 13 (1), 2004. p. 13-24.

MCENERY, Tony and HARDIE, Andrew. Corpus linguistics: method, theory and practice. Cambridge: Cambridge University Press, 2012, p. 1-24. 
SALDANHA, Gabriela. Style of Translation: An exploration of stylistic patterns in the translations of Margaret Jull Costa and Peter Bush. Tese, Dublin City University, Dublin, 2005. 25-50, 2011.

. Translator style: methodological considerations. The Translator, v. 17, n. 1, p.

. Style of Translation: The use of foreign words in translations by Margaret Jull Costa e Peter Bush. In: KRUGER, Alet; WALMACH, Kim; MUNDAY, Jeremy (Eds.) Corpus Based Translation Studies Research and Applications. London \& New York: Bloomsbury, 2011, p. 237-258.

\footnotetext{
${ }^{1}$ Currículo lattes Cristina Lazzerini de Souza. Disponível em: http://lattes.cnpq.br/5164871387903855

2 No original: [...] "a consistent and statistically significant regularity of occurrence in text of certain items and structures, or types of items and structures, among those offered by the language as a whole.”

3 No original:

- a mediated text is affected by the mediator's interpretation of the original;

- mediation through translation always has a purpose;

- the purpose the translation is intended to serve may differ from the purpose the original text was intended to serve;

52 the audience for the translation is almost always different from the audience for the original text.

${ }^{4}$ No original: $[\ldots]$ "how the text means what it does” [...]

${ }^{5}$ No original: [...] "explain why, given the source text, the translation has been shaped in such a way that it comes to mean what it does”.
}

RECEBIDO EM: 26 de março de 2015

ACEITO EM: 01 de julho de 2015 